\title{
Design of Intelligent Self-Tuning GA ANFIS Temperature Controller for Plastic Extrusion System
}

\author{
S. Ravi, ${ }^{1}$ M. Sudha, ${ }^{2}$ and P. A. Balakrishnan ${ }^{3}$ \\ ${ }^{1}$ Department of EEE, Nandha Engineering College, Erode 638052, Tamilnadu, India \\ ${ }^{2}$ Department of ECE, Karpagam Institute of Technology, Coimbatore 641105, Tamilnadu, India \\ ${ }^{3}$ K.C.G. College of Technology, Chennai 600097, Tamilnadu, India
}

Correspondence should be addressed to S. Ravi, toravi2003@gmail.com

Received 19 October 2010; Revised 18 April 2011; Accepted 22 May 2011

Academic Editor: A. Mohamed

Copyright (C) 2011 S. Ravi et al. This is an open access article distributed under the Creative Commons Attribution License, which permits unrestricted use, distribution, and reproduction in any medium, provided the original work is properly cited.

\begin{abstract}
This paper develops a GA ANFIS controller design method for temperature control in plastic extrusion system. Temperature control of plastic extrusion system suffers problems related to longer settling time, couple effects, large time constants, and undesirable overshoot. The system is generally nonlinear and the temperature of the plastic extrusion system may vary over a wide range of disturbances. The system is designed with three controllers. The proposed GA ANFIS controller is the most powerful approach to retrieve the adaptiveness in the case of nonlinear system. In this research the control methods are simulated using simulink. Relatively the methodology and efficiency of the proposed method are compared with those of the traditional methods and the results obtained from GA ANFIS controller give improved performance in terms of time domain specification, set point tracking, and disturbance rejection with optimum stability.
\end{abstract}

\section{Introduction}

The temperature control in plastic extrusion machine is an important factor to produce high-quality products. Plastic extrusion is a well-known process and widely used in polymerization industry. The extrusion consists of large barrel divided into three temperature zones, namely, barrel, adapter, and die zone, respectively. The temperature zone uses more number of heaters in order to provide different temperature ranges. The overall structure of the plastic extrusion is shown in Figure 1. The polymer is fed into the hopper in solid pellet forms and it passes through the temperature zones where it is heated and melted. The melted polymer material is pushed forward by a powerful screw and it passes through the molding mechanism from the die. The quality of extrudates depends on uniform temperature distribution, physical property of raw material, and so forth. The temperature section of PVC extrusion plant is shown in Figure 2. High efficient plastic extrudates can be obtained only when temperature in all the zones is precisely controlled [1]. The implementation of PID controllers retunes their three-term parameters so as to ensure that the dynamic behavior of extruder performance is satisfactory along with the specific heat, thermal conductivity, and ambient temperature which vary with time. PID controllers are used for almost all industrial processes. However, PID controller performs well only at a particular operating range and it is necessary to retune the PID controller if the operating range is changed. The PID controllers do not provide contented results for nonlinear and dead time process [2].

In addition with that, the flow of heat from one temperature zone to another may cause bad transient response for the heating process under set point and load variation. The difficult task of modeling and controlling complex real world systems is difficult especially when implementation issues are considered. If a relatively accurate model of a dynamic system can be developed, it is often too complex to use in controller development, especially because many conventional control design techniques require restrictive assumptions, for the plant model and for the control to be designed (e.g., linearity). Not taking into account these assumptions result in a number of unknown variables which the controller design techniques are unable to handle. This is because process industry machines, unlike humans, lack 




FIgURE 1: Overall structure of the plastic extrusion system.

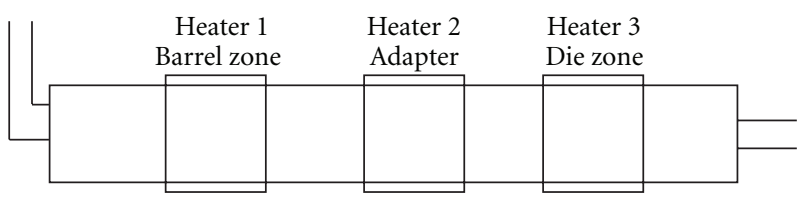

Figure 2: Temperature section of PVC plant.

the ability to solve problems using imprecise information. To emulate this ability fuzzy logic and fuzzy sets are introduced. Fuzzy controllers are not like PID; they are robust. Their performances are less sensitive to parametric variations. The fuzzy controller can be designed without knowing the mathematical model of system. Fuzzy logic controllers have been reported successful for a number of complex and nonlinear processes [3]. Fuzzy can operate for wide range and is capable of maintaining set point temperature levels and reducing overshoots. The genetic algorithm-based neurofuzzy controller has the integral advantages of neural and fuzzy approaches and they are used for intelligent decision making systems. Genetic algorithm uses a direct analogy of such natural evolution to do global optimization in order to solve highly complex problems. It presumes that the potential solution of a problem is individual and can be represented by a set of parameters. Neural networks and fuzzy logic represent two distinct methodologies to deal with uncertainty. Neural networks can model complex nonlinear relationships and are quietably suited for classification phenomenon of predetermined classes. The output is not limited to zero error but minimization of least square errors occurs. Training time required is large for neural network [4]. Training data has to be chosen carefully, to cover the entire range over which different variables are expected. Neural networks and fuzzy logic are different technologies which can be used to accomplish the specification of mathematical relationships. Among numerous variables in a complex dynamic process, these perform mappings with some degree of imprecision in different ways which are used to control nonlinear systems. Hence by strengthening the neurofuzzy controllers with genetic algorithms the searching and attainment of optimal solutions will be easier and faster. The benefits of harnessing the capabilities of genetic algorithms are huge, research efforts on optimizing the solutions are challenging. The combination of genetic algorithm and neuro fuzzy controllers is normally shortened as GAANFIS and this intelligent hybrid controller is compared with that of the conventional PID and fuzzy controller. The Matlab/Simulink software forms part of the modeling and design tool employed in this research.

\section{Temperature System in Plastic Extrusion Model}

Step response method is based on transient response tests. Many industrial processes have step responses of the system in which the step response is monotonous after an initial time. A system with step response can be approximated by the transfer function as in (1) where " $k$ " is the static gain, " $\tau$ " is the apparent time delay, and " $T$ " is the apparent time constant. $G(s)$ is the transfer function of the plant. The transfer function of plastic extrusion pipeline described is given in (2), the plastic extrusion model uses the parameters $k=0.92, T=144$ seconds, $\tau=10$ seconds [5], and the temperature generally varies from $50^{\circ} \mathrm{C}$ to $200^{\circ} \mathrm{C}$ :

$$
\begin{gathered}
G(s)=\frac{k}{1+s T} e^{-s \tau}, \\
G(s)=\frac{0.92}{1+144 s} e^{-10 s} .
\end{gathered}
$$

\section{PID Control}

The PID control is designed to ensure the specifying desired nominal operating point for temperature control of plastic extrusion model and regulating it, so that it stays closer to the nominal operating point in the case of sudden disturbances, set point variations, and noise. The proportional gain $\left(K_{p}\right)$, 


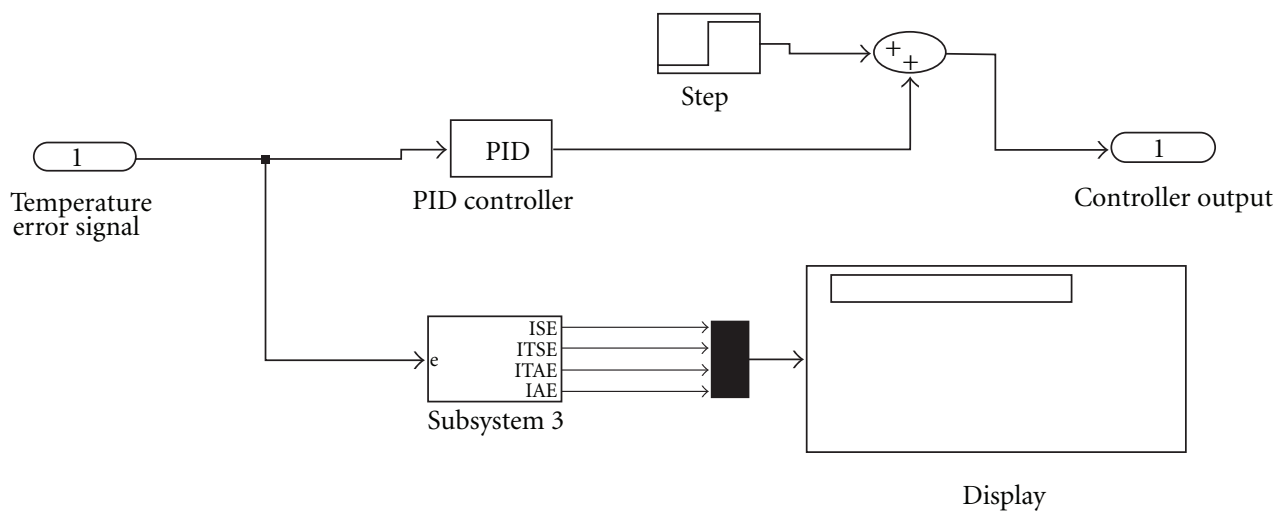

FIgURE 3: Simulink model of PID controller.

TABLE 1: Ziegler-Nichols tuning rules.

\begin{tabular}{lccc}
\hline Type of controller & $K_{p}$ & $T_{i}$ & $T_{d}$ \\
\hline $\mathrm{P}$ & $T / L$ & $\infty$ & 0 \\
$\mathrm{PI}$ & $0.9 T / L$ & $L / 0.3$ & 0 \\
$\mathrm{PID}$ & $1.2 T / L$ & $2 L$ & $0.5 L$ \\
\hline
\end{tabular}

TABLE 2: Minimum setting values of ISE, ITSE, ITAE, and IAE.

\begin{tabular}{lccc}
\hline $\begin{array}{l}\text { Integral square } \\
\text { error }\end{array}$ & $\begin{array}{c}\text { Integral time } \\
\text { square error }\end{array}$ & $\begin{array}{c}\text { Integral time } \\
\text { average error }\end{array}$ & $\begin{array}{c}\text { Integral } \\
\text { average error }\end{array}$ \\
\hline $8.054 \mathrm{e}+6$ & $5.971 \mathrm{e}+10$ & $1.962 \mathrm{e}+9$ & $2.768 \mathrm{e}+5$ \\
\hline
\end{tabular}

Table 3: Minimum setting values $K_{p}, K_{i}$, and $K_{d}$.

\begin{tabular}{ccccc}
\hline$K_{p}$ & $T_{i}(\mathrm{~s})$ & $T_{d}$ & $K_{i}$ & $K_{d}$ \\
\hline 30 & 20 & 05 & $0.315 \mathrm{e}+10$ & 11.52 \\
\hline
\end{tabular}

TABLe 4: Proposed fuzzy rules.

\begin{tabular}{lccccc}
\hline$e$ & & & $c e$ & & \\
& NB & NS & Z & PS & PB \\
\hline NB & NB & NB & NB & NS & Z \\
NS & NB & NS & NS & Z & PS \\
Z & NB & NS & Z & PS & PB \\
PS & NS & Z & PS & PS & PB \\
PB & Z & PS & PB & PB & PB \\
\hline
\end{tabular}

integral time constant $\left(T_{i}\right)$, and derivative time constant $\left(T_{d}\right)$ of the PID control settings are designed using ZeiglerNichols tuning method as shown in Table 1. By applying the step test to (1) the S-shaped curve is obtained and there is identified the temperature control method characterized by two constants as delay time $L=10$ seconds and time constant $=50$ seconds. The delay time and time constant are determined by drawing a tangent line at the inflection point of the S-shaped curve and determined by the intersections of the tangent line with the time axis and line output response $c(t)$. From Zeigler-Nicholas tuning rule, the suggested optimal set $\left(K_{p}\right)$, and $\left(T_{i}\right),\left(T_{d}\right)$ values are obtained [6]. The optimal setting values $\left(K_{p}\right),\left(T_{i}\right)$, and $\left(T_{d}\right)$ obtained for temperature control of plastic extrusion model are obtained by finding the minimum values of integral square error, integral time square error, integral time average error, and integral average error shown in Table 2. The minimum setting values of $K_{p}, K_{i}$, and $K_{d}$ shown in Table 3. The simulink model of block of PID control is shown in Figure 3.

\section{Fuzzy Controller and Its Membership Function}

Fuzzy logic is more effective feedback control system and easier to implement. Fuzzy controller consists of a fuzzifier, rule base, an inference engine, and a defuzzifier [7]. The numerical input values of the fuzzifier are converted into fuzzy values, along with the rule base which are fed into the inference engine which produces control value. In fuzzy rule base, various rules are fostered according to their respective problem requirements. The control values are not in usable form; henceforth they are converted to numerical output values using the defuzzifier. The plastic extrusion temperature controller uses two-dimensional fuzzy controller models which are shown in Figure 4. It has two input variables, error " $e$ ", change in error "ce", and one output variable " $u$ ". For computations to be relatively simple, the research uses triangular membership function. The computational structure of FLC scheme is composed of the steps rule base and membership function. The fuzzy control rules were formulated in the IF-THEN rules form. The rule base stores the rules governing the input and output relationship of proposed control logic [8]. The inputs to the fuzzy controller error $e(k)$ and change in error $\Delta e(k)$ computed from the reference value $r(k)$. The $k$ denotes the discrete time. The fuzzy controller output $u(k)$ is based on error and error change. Table 4 summarizes the 25 rules for the proposed control algorithm for fuzzy logic. Each universe of discourse is divided into five fuzzy subsystems, namely, NB, NS, Z, PS, and $\mathrm{PB}$. The input and change in input variable $(e, c e)$ are shown in Figures 5 and 6 . The inference mechanism is used for evaluating linguistic descriptions. The fuzzy control rules have been described using linguistic variables; for example, if error $e$ is NS and the increasing change in error $c e$ is $\mathrm{PB}$, then 


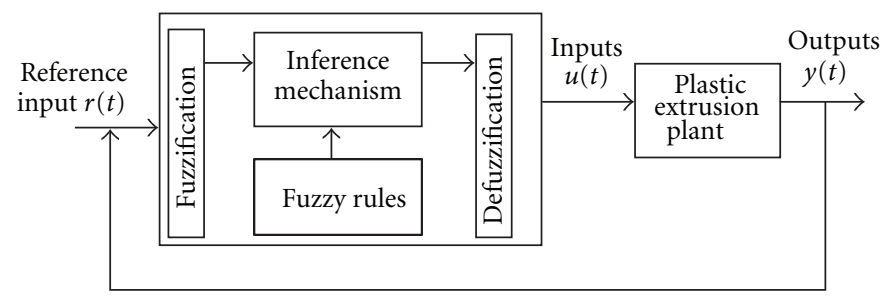

FIGURE 4: FLC controller-based plastic extrusion system.

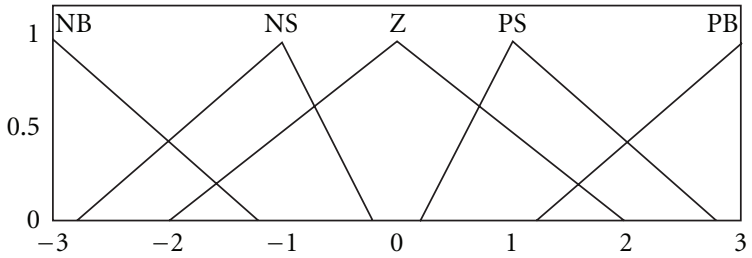

Figure 5: Fuzzy controller input variable “e”.

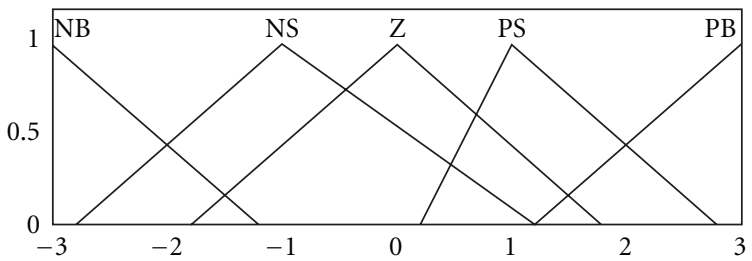

Figure 6: Fuzzy controller input variable “ce”.

the output is PS which is used to control the temperature rise. The output variable of fuzzy set $u$ is shown in Figure 7.

The inference result of each rule consists of two parts, the weighing factor $w_{i}$ of the individual rule and the degree of change of temperature $C$. According to the rule, it is written as follow:

$$
z_{i}=\min \left(\mu_{e}\left(e_{0}\right), \mu_{c e}\left(c e_{0}\right)\right), \quad C_{i}=w_{i} C_{i},
$$

where $z_{i}$ denotes the change in control signal inferred by the $i$ th rule and $C$ is noted from the rule table, which shows the mapping from the product space of $e$ and $c e$ to $C_{i}[9,10]$.

The defuzzification process is after collecting all the singleton rules; it defuzzifies the result so that a crisp value control signal is obtained and the change of the control signal is computed using center of gravity method as given in (4). The simulink model block of fuzzy control is shown in Figure 8:

$$
z=\delta u_{k}=\frac{\sum_{i=1}^{N} z_{i}}{\sum_{i=1}^{N} w_{i}}
$$

\section{GA ANFIS Model}

The genetic algorithm technique employed to tune the ANFIS controller. Genetic algorithm was inspired by the mechanism of natural selection, a biological process in which stronger individual is likely to be the winners in

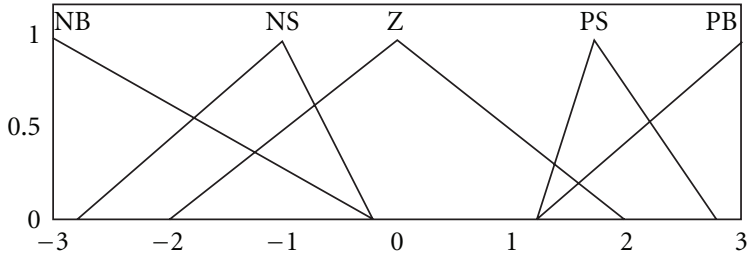

Figure 7: Fuzzy controller output variable " $u$ ”.

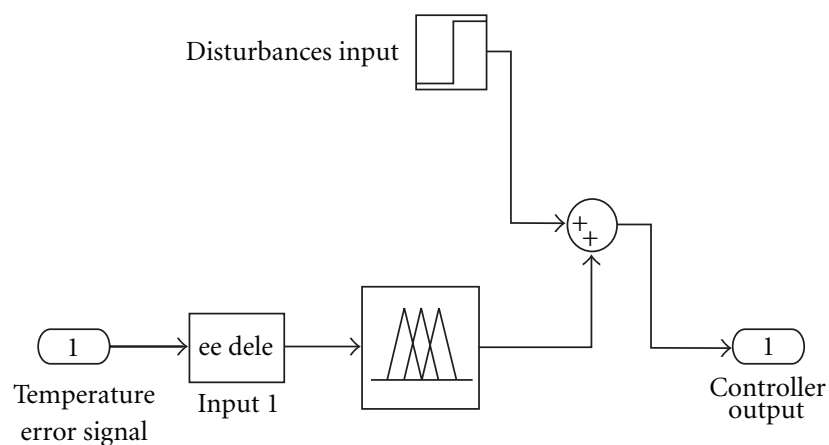

FIGURE 8: Simulink model of fuzzy controller.

a competing environment. Genetic algorithm uses a direct analogy of such natural evolution to do global optimization in order to solve highly complex problems. It presumes that the potential solution of a problem is individual and can be represented by a set of parameters. These parameters are regarded as the genes of a chromosome and can be structured by a string of concatenated values. The form of variables representation is defined by the encoding scheme. The variables can be represented by binary, real numbers, or other forms, depending on the application data. Its range, the search space, is usually defined by the problem. Genetic algorithm has been successfully applied to many different problems. The tuning approach employs the use of matlab M-files and functions to manipulate the ANFIS system and scaling gains, run the simulink-based simulation, checking the resulting performance, and continuously modify the system for a number of times in search for optimal solution. The GA optimization algorithm was run for 1000 epochs. Fuzzy logic and neural networks are natural complementary tools in building intelligent systems. Neural networks are computational structures that perform well, when dealing with new data, while fuzzy logic deals with reasoning, using linguistic information acquired from domain experts. Fuzzy 


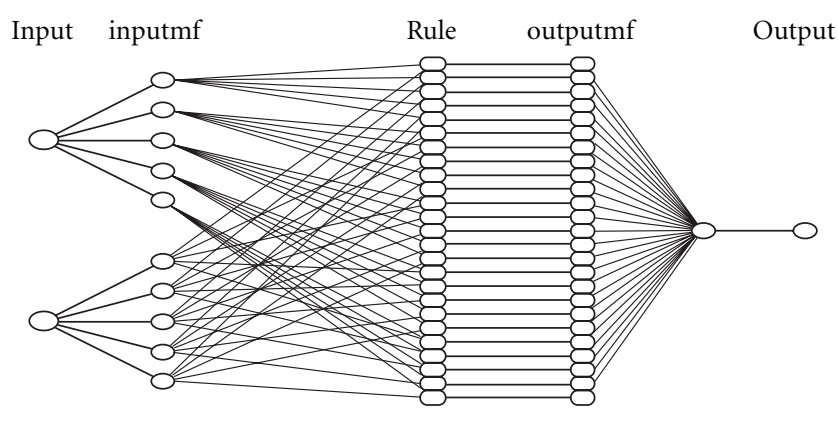

FIgURE 9: Internal layer of GA ANFIS model.

systems lack the ability to learn and cannot adjust themselves to a new environment. Neural networks can learn and they are opaque to the user. The neural network merged with a fuzzy system, forms one integrated system. It offers a promising approach to build intelligent systems. Integrated systems can combine the parallel computation and learning abilities of neural networks, with the human knowledge representation and explanation abilities of fuzzy systems. The neural network, uses feed forward network; the number of input and output layers used for this system is one with linear saturation function. The hidden layer used for this system is two and using tansigmoidal function. Fuzzy inference systems are also known as fuzzy rule-based systems, containing a number of fuzzy IF-THEN rules. GA ANFIS is used in the form of Takaji sugeno model to integrate the best features of fuzzy systems and neural networks. GA ANFIS is also used in representation of prior knowledge into a set of constraints, to reduce optimization search space obtained from fuzzy and adaptation of back propagation to structured network through neural network [11, 12]. To train the GA ANFIS controller generally input-output characterization or desired output of the plant is sufficient. For better performance two systems hybridized. The error and derivative error are given as an input to the system and the neural network's output is given to the fuzzy logic. Neural network will decide which fuzzy set is selected out of five fuzzy sets. The maximum membership set is selected. The genetic learning algorithm tunes the membership functions of a Sugeno type fuzzy inference system using the training input-output data. These modeling methods can be applied to both static and dynamic systems. If the output of the model at a moment is applied as its input at the next moment, the model is called dynamic model or recurrent model. In other words, in recurrent models, the output of the model at the existing moment is influenced by the output of the model, at previous moments. The GA ANFIS system rule base is shown in Table 5 . The proposed algorithm summarizes 25 rules. In a GA ANFIS controller training algorithm, each epoch is composed of a forward pass and backward pass [13]. In the forward pass, a training set of input patterns is presented to the GA ANFIS controller, the neurons outputs are calculated on the layer- by-layer basis, and the rules consequent parameters are identified by the least squares estimator. The GA ANFIS system consists of the components of a conventional fuzzy system. But, these computations at each stage are performed by hidden neurons
TABLE 5: Proposed GA ANFIS control rules.

\begin{tabular}{lccccc}
\hline$e$ & & \multicolumn{5}{c}{$c e$} \\
& NB & NS & Z & PS & PB \\
\hline NB & MF1 & MF2 & MF3 & MF4 & MP5 \\
NS & MF6 & MF7 & MF8 & MF9 & MF10 \\
Z & MF11 & MF12 & MF13 & MF14 & MF15 \\
PS & MF16 & MF17 & MF18 & MF19 & MF20 \\
PB & MF21 & MF22 & MF23 & MF24 & MF25 \\
\hline
\end{tabular}

and the neural network learning capacity is provided to enhance the system knowledge [14]. The multi-layer fuzzy neural network model for fuzzy tuning rules is given in Figure 9. It shows the diagram for the internal layers of ANFIS model. The optimal value of the neuro fuzzy controller is found by using genetic algorithm. All possible sets of controller parameter values are particles whose values are adjusted to minimize the objective function. For the GA ANFIS controller design, it is ensured that the controller settings estimated result in a stable closed loop system.

5.1. Initialization of Parameters. To start with genetic algorithm, certain parameters need to be defined. These include population size, bit length of chromosome, number of iterations, selection, crossover, and mutation types. Selection of these parameters decides, to a great extend, the ability of the designed controller. The range of the tuning parameters is considered between 0 and 10. Initializing values are detailed as follows:

(i) population type: double vector,

(ii) selection function: tournament selection,

(iii) tournament size: 2 ,

(iv) reproduction crossover function: 0.8 ,

(v) crossover function: scattered,

(vi) migration direction: forward,

(vii) mutation function: constraint dependent default value.

In each generation, the genetic operators are applied to selected individuals from the current population in order to create a new population. Generally, the three main genetic operators of reproduction, crossover, and mutation are employed. By using different probabilities for applying these operators, the speed of convergence can be controlled. Crossover and mutation operators must be carefully designed, since their choice highly contributes to the performance of the whole genetic algorithm [15].

Reproduction. A part of the new population can be created by simply copying without changing selected individuals from the present population. Also new population has the possibility of selection by already developed solutions. There are a number of other selection methods available and it is up to the user to select the appropriate one for each process. Reproduction crossover fraction is using 0.8 . 


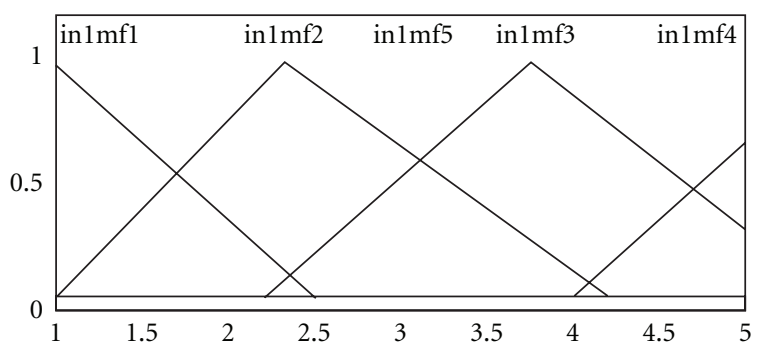

FIGURE 10: GA ANFIS controller input variable " $e$ ".

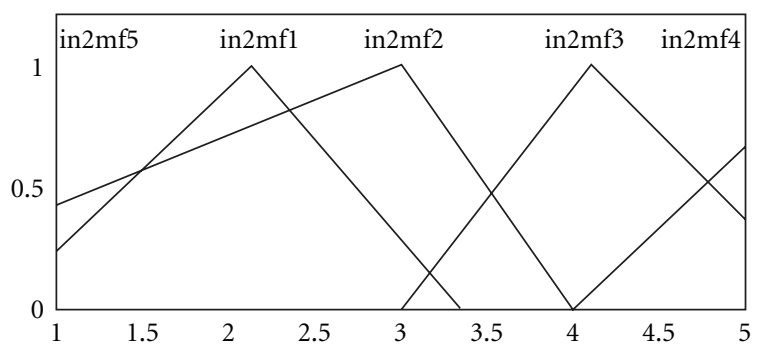

FIGURE 11: GA ANFIS controller input variable " $c e$ ".

Crossover. New individuals are generally created as offspring of two parents (i.e., crossover being a binary operator). One or more so-called crossover points are selected (usually at random) within the chromosome of each parent, at the same place in each. The parts delimited by the crossover points are then interchanged between the parents. The individuals resulting in this way are the offspring. Beyond one point and multiple point crossovers, there exist some crossover types. The so-called arithmetic crossover generates an offspring as a component-wise linear combination of the parents in later phases of evolution, it is more desirable to keep individuals intact, and so it is a good idea to use an adaptively changing crossover rate: higher rates in early phases and a lower rate at the end of the genetic algorithm.

Mutation. A new individual is created by making modifications to one selected individual. The modifications can consist of changing one or more values in the representation or adding/deleting parts of the representation. In genetic algorithm, mutation is a source of variability and too great a mutation rate results in less efficient evolution, except in the case of particularly simple problems. Hence, mutation should be used sparingly because it is a random search operator; otherwise, with high mutation rates, the algorithm will become little more than a random search. Moreover, at different stages, one may use different mutation operators. At the beginning, mutation operators resulting in bigger jumps in the search space might be preferred [16]. Later on, when the solution is close by a mutation operator leading to slighter shifts in the search space could be favored. Figures 10 and 11 show the input and change in input variable $(e, c e)$ of GA ANFIS controller. The output variable of fuzzy set " $u$ " is shown in Figure 12. The simulink model block of GA ANFIS controller section is shown in Figure 13.

$\begin{array}{ll}\text { out1mf13 } & \text { out1mf25 } \\ \text { out1mf12 } & \text { out1mf24 } \\ \text { out1mf11 } & \text { out1mf23 } \\ \text { out1mf10 } & \text { out1mf22 } \\ \text { out1mf9 } & \text { out1mf21 } \\ \text { out1mf8 } & \text { out1mf20 } \\ \text { out1mf7 } & \text { out1mf19 } \\ \text { out1mf6 } & \text { out1mf18 } \\ \text { out1mf5 } & \text { out1mf17 } \\ \text { out1mf4 } & \text { out1mf16 } \\ \text { out1mf3 } & \text { out1mf15 } \\ \text { out1mf2 } & \text { out1mf14 } \\ \text { out1mf1 } & \end{array}$

FIGURE 12: GA ANFIS controller output variable " $u$ ".

\section{Simulation Results}

The system is a multistage and coupled system; so, four set points are taken for the system. The Matlab simulink used for simulation and uses of the first-order function. Four set point temperatures $70^{\circ} \mathrm{C}, 100^{\circ} \mathrm{C}, 150^{\circ} \mathrm{C}$, and $200^{\circ} \mathrm{C}$ changes at different times are used in 14000 seconds. The results of PID controller for temperature set point shown in Figure 14 and it is observed that the performance of the system with PID controller is almost oscillating and takes more time to settle with reference temperature, compared with other types of controller. The fuzzy simulation output diagram for fuzzy is given in Figure 15. FLC is designed using 5 linguistic levels and 25 rules. FLC gives better results compared to PID controller by giving a quick settle with reference temperature and less oscillatory response. The integrated GA ANFIS output controller output with Takaji Sugeno fuzzy model is shown in Figure 16 which eliminates the oscillatory output of different temperature set points and identifies the process variation quickly and provides good control for set point changes and sudden disturbances. The different controllers PID, FLC, and proposed GA ANFIS controller output are depicted in Figure 17. The results shows that the proposed controller output settles with reference temperature very quickly and it eliminates the overshoot problem. The result of the GA-optimized ANFIS controller shows an outstanding performance in terms of achieving the desired value with very small values for the rise time and settling time.

\section{Results and Discussion}

In this paper, GA ANFIS controller acts as a replacement for the previous existing controllers due to its unique characteristics. The merits of the GA ANFIS can be observed as follows. The GA ANFIS has improved control quality. The aim of controlling heated barrel is to bring the set points during startup as soon as possible, while avoiding large 




FIgURE 13: Simulink model of GA ANFIS controller.

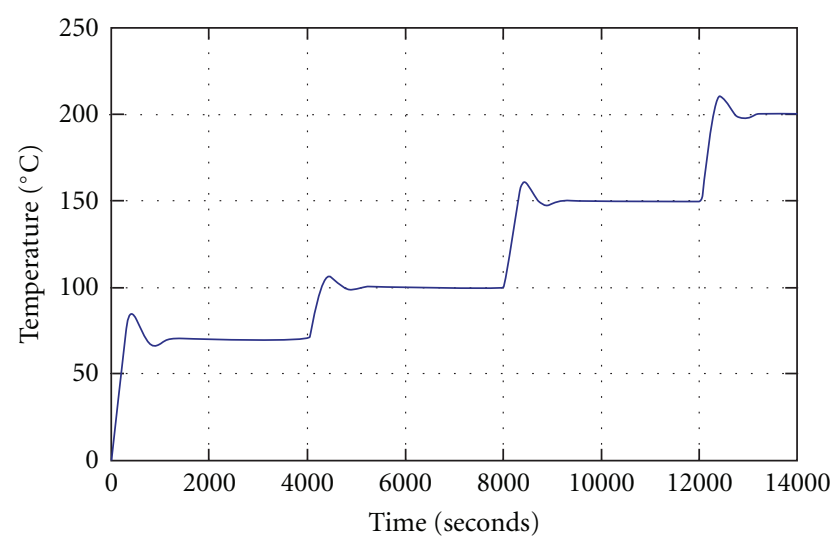

FIGURE 14: PID control simulated output at different temperature set points.

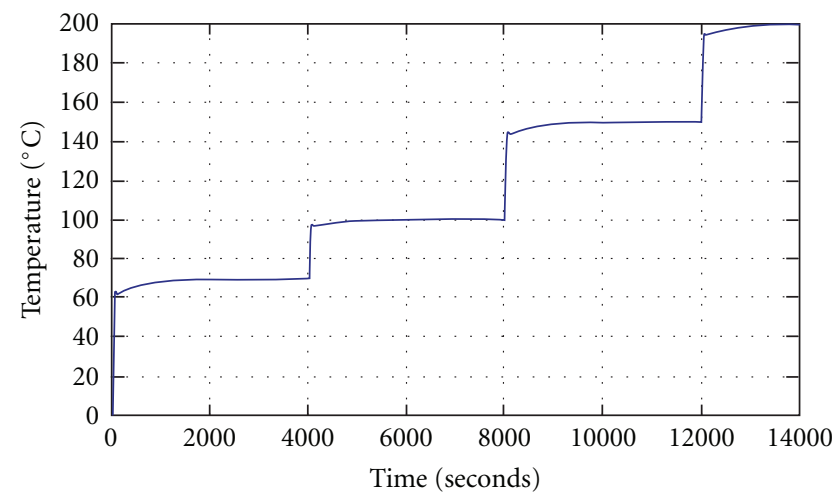

FIGURE 15: FLC control simulated output at different temperature set points.

overshoots in order to maintain it at current temperature set value. Table 6 gives various timing specification for the three controllers. From the analysis, the GA ANFIS controller on the basis of delay time gives efficient output differences 8.5 times as that of PID controller and 1.5 times as that of fuzzy. Consequently, the GA ANFIS controller produces an output, which is 5 times ahead of PID and 1.6 times of fuzzy, in the rise time analysis. The peak time results state that the GA ANFIS controller outcasts a production output 6.66 times more efficient than PID and 1.66 times than fuzzy. If we consider the settling time, the GA ANFIS controller is 1.15 times more efficient than PID and 1.09 times than fuzzy. The set points tracking and disturbance rejection are obtained by the proposed method.

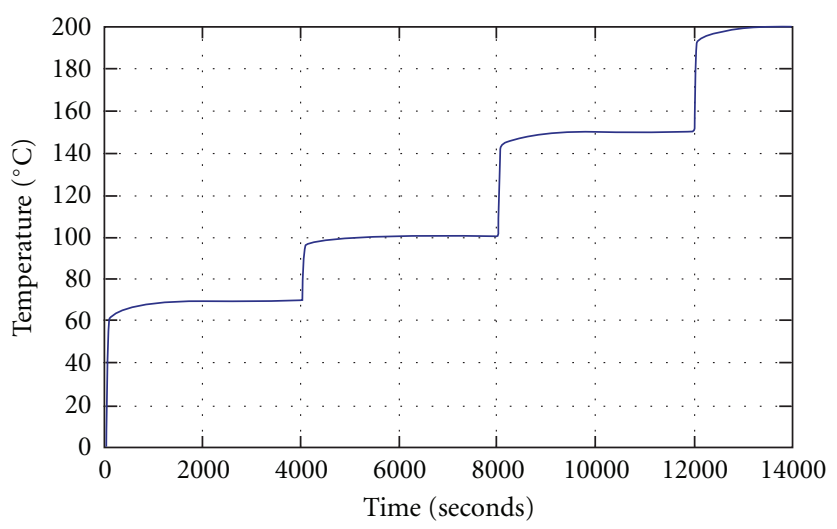

Figure 16: GA ANFIS controller simulated output at different temperature set points.

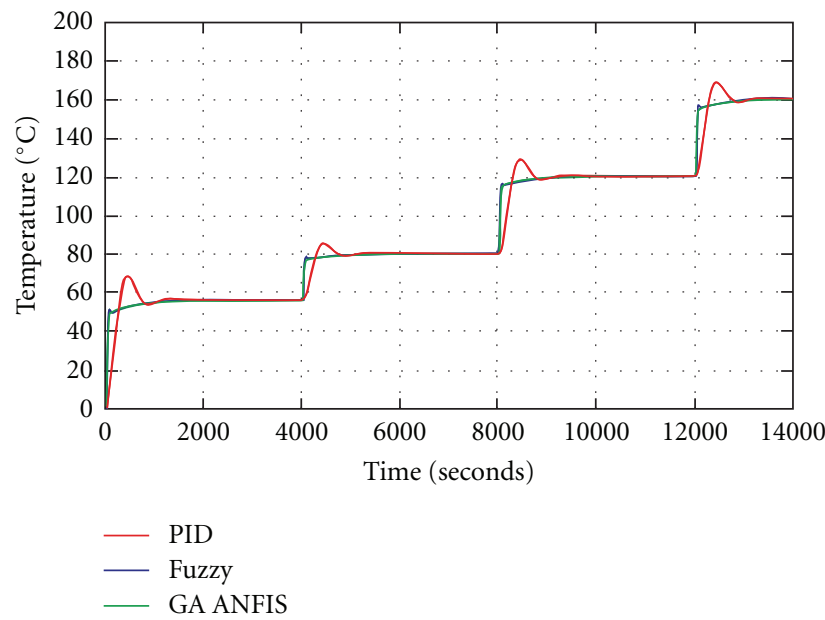

Figure 17: Comparison results of PID, FLC, and GA ANFIS controllers.

\section{Conclusion}

We have chosen GA ANFIS controller, since it is characterized by its capability to eliminate sudden input disturbance and maintain the set point temperature in the plastic extrusion system. The simulation results clearly show that the GA ANFIS controller reduces the timing specifications of fuzzy and PID controllers. This paper demonstrates the effectiveness of intelligent controller on nonlinear system, particularly for temperature control in plastic extrusion system. The comparison of performance of the three controllers 
TABle 6: Timing specification of PID, FLC, and GA ANFIS controllers.

\begin{tabular}{lccc}
\hline Timing specifications & PID & FUZZY & GA ANFIS \\
\hline Delay time $\left(T_{d}\right)$ & $170 \mathrm{Sec}$ & $25 \mathrm{Sec}$ & $20 \mathrm{Sec}$ \\
Rise time $\left(T_{r}\right)$ & $250 \mathrm{Sec}$ & $80 \mathrm{Sec}$ & $50 \mathrm{Sec}$ \\
Peak time $\left(T_{p}\right)$ & $400 \mathrm{Sec}$ & $100 \mathrm{Sec}$ & $60 \mathrm{Sec}$ \\
Settling time $\left(T_{s}\right)$ & $1900 \mathrm{Sec}$ & $1800 \mathrm{Sec}$ & $1650 \mathrm{Sec}$ \\
Peak overshoot $(\%)$ & $21 \%$ & 0 & 0 \\
\hline
\end{tabular}

reveals that the GA ANFIS controller is superior to the other controllers. From the results obtained the proposed controller is good for set point changes and stability. With the aid of the supervisory technique, the proposed controller identifies the process variations quickly and provides good controller performance for the set point changes and sudden disturbances. Therefore GA ANFIS controller will prove efficacious especially in the case of plastic extrusion temperature control system.

\section{References}

[1] H. Shu and Y. Pi, "Decoupled temperature control system based on PID neural network," in Proceedings of the Automatic Control and System Engineering Conference (ACSE '05), Cairo, Egypt, 2005.

[2] C.-C. Tsai and C.-H. Lu, "Fuzzy supervisory predictive pid control of a plastics extruder barrel," Journal of the Chinese Institute of Engineers, vol. 21, no. 5, pp. 619-624, 1998.

[3] M. N. Ab Malek and M. S. M. Ali, "Evolutionary tuning method for PID controller parameters of a cruise control system using metamodeling," Modelling and Simulation in Engineering, vol. 2009, Article ID 234529, 8 pages, 2009.

[4] T. Korkobi, M. Djemel, and M. Chtourou, "Stability analysis of neural networks-based system identification," Modelling and Simulation in Engineering, vol. 2008, Article ID 343940, 8 pages, 2008.

[5] H. Zhou, "Simulation on temperature fuzzy control in injection mould machine by Simulink," Asian Journal of Control, vol. 5, pp. 176-186, 2003.

[6] M. Y. Hassan and W. F. Sharif, "Design of FPGA based PID like fuzzy controller for industrial applications," IAENG International Journal of Computer Science, vol. 34, no. 2, pp. 192-198, 2007.

[7] C. F. Juang, S. T. Huang, and F. B. Duh, "Mold temperature control of a rubber injection-molding machine by TSK-type recurrent neural fuzzy network," Neurocomputing, vol. 70, no. 1-3, pp. 559-567, 2006.

[8] C.-C. Tsai and C.-H. Lu, "Multivariable self-tuning temperature control for plastic injection molding process," IEEE Transactions on Industry Applications, vol. 34, no. 2, pp. 310 $318,1998$.

[9] C.-F. Juang and C.-H. Hsu, "Temperature control by chipimplemented adaptive recurrent fuzzy controller designed by evolutionary algorithm," IEEE Transactions on Circuits and Systems-I, vol. 52, no. 11, pp. 2376-2384, 2005.

[10] P. P. Bhogle, B. M. Patre, L. M. Waghmare, and V. M. Panchade, "Neuro fuzzy temperature controller," in Proceeding of The International Conference on Mechatronics and Automation (IEEE '07), pp. 3344-3348, Harbin, China, 2007.
[11] J.-H. Lai and C.-T. Lin, "Application of neural fuzzy network to pyrometer correction and temperature control in rapid thermal processing," IEEE Transactions on Fuzzy Systems, vol. 7, no. 2, pp. 160-175, 1999.

[12] C. F. Juang and C. T. Lin, "A recurrent self-organizing neural fuzzy inference network," IEEE Transactions on Neural Networks, vol. 10, no. 4, pp. 828-845, 1999.

[13] I. Yusuf, N. Iksan, and A. S. Herman, "A temperature control for plastic extruder used fuzzy genetic algorithms," in Proceedings of the International MultiConference of Engineers and Computer Scientists (IMECS '10), pp. 1075-1080, Hong Kong, 2010.

[14] G. Saravanakumar and R. S. D. WahidaBanu, "An adaptive controller based on system identification for plants with uncertainties using well known tuning formulas," International Journal of Automatic Control and System Engineering, vol. 6, no. 3, pp. 17-22, 2006.

[15] S. Nara, P. Khatri, and J. Garg, "Proportional integral derative controller tuning of temperature control system using genetic algorithm," International Journal Of Electronics, Information and Systems, vol. 12, no. 2, pp. 37-42, 2010.

[16] S. M. G. Kumar, R. Jain, and N. Anantharaman, "Genetic algorithm based PID controller tuning for a model bioreactor," Indian Chemical Engineer, vol. 50, no. 3, pp. 214-226, 2008. 

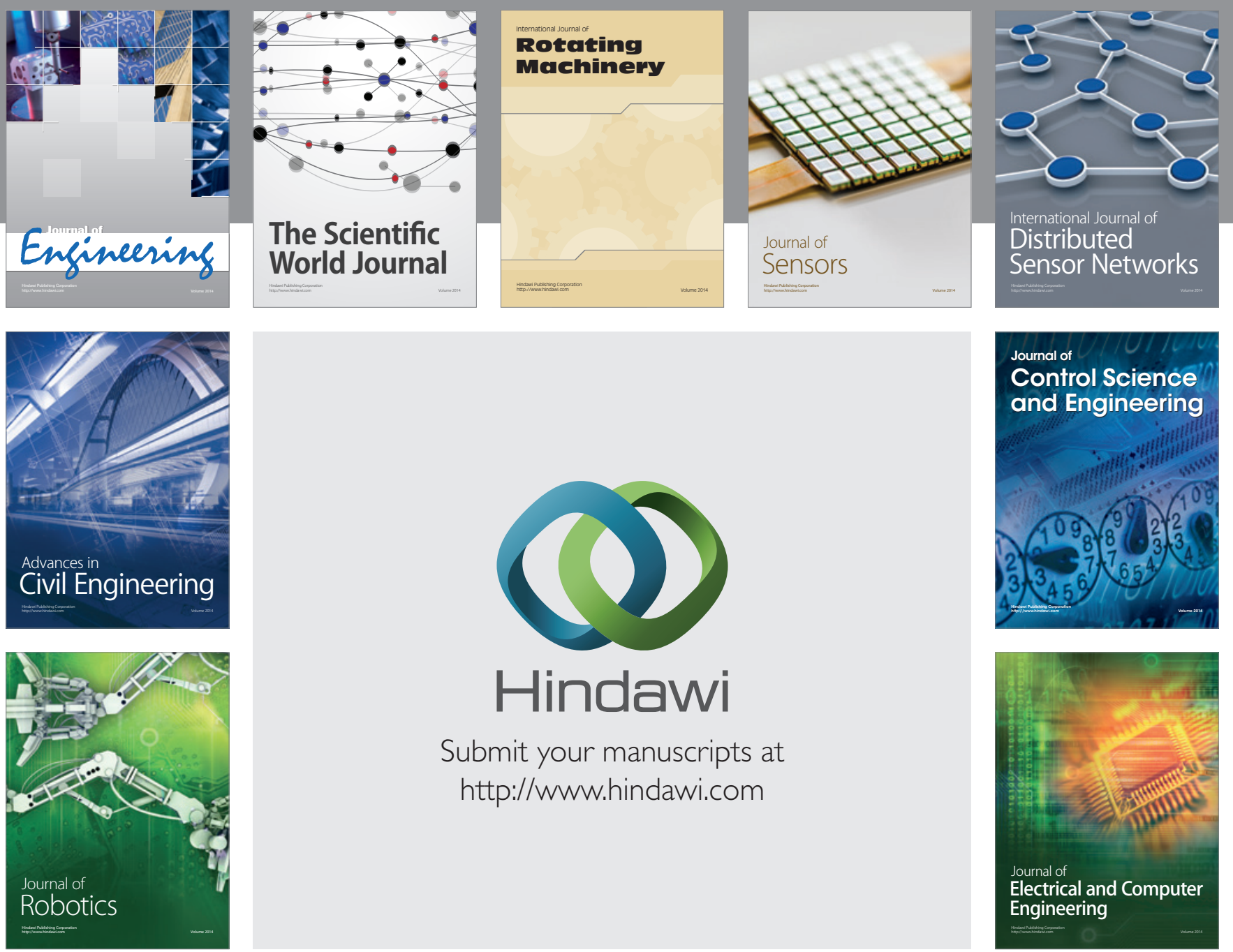

Submit your manuscripts at

http://www.hindawi.com
